International Journal of Algebra, Vol. 1, 2007, no. 7, 335 - 346

\title{
W-type and H-type Non-Associative Algebras using Additive Maps I
}

\author{
Seul Hee Choi \\ Dept. of Mathematics, Univ. of Jeonju \\ Chon-ju 560-759, Korea \\ choi@jj.ac.kr \\ Jeong-Sig Lee \\ Department of General Studies and Education \\ Sahmyook Univ., Cheongnyangni \\ P.O. Box 118, 130-650, Seoul, Korea \\ toplee@syu.ac.kr \\ Ki-Bong Nam \\ Department of Mathematics \\ University of Wisconsin-Whitewater \\ Whitewater, WI 53190, USA \\ namk@uww.edu
}

\begin{abstract}
A $W$-type Lie algebra is defined using a map from an additive group to a field of characteristic zero in the paper (see [10]). We define Lieadmissible $W$-type and $H$-type non-associative algebras over a field of any characteristic in this work (see [9]). We show that those algebras are simple.
\end{abstract}

Keywords: simple, $W$-type algebra, $H$-type algebra, graded algebra

\section{Preliminaries}

It is an interesting problem to find a simple algebra (see [6]). Let $\mathbb{F}$ be a field of characteristic zero and $\mathbb{F}_{p}$ a field of characteristic $p$. Throughout this paper, 
$\mathbb{N}$ and $\mathbb{Z}$ will denote the non-negative integers and the integers, respectively. For a given algebra $A$, we can define its anti-symmetric algebra $A^{-}$using the commutator [,] (see [1], [10], and [11]). The $W$-type non-associative algebra $N W(n)$ has the standard basis

$$
\left\{x_{1}^{a_{1}} \cdots x_{n}^{a_{n}} \partial_{u} \mid a_{1}, \cdots, a_{n} \in \mathbb{N}, 1 \leq u \leq n\right\}
$$

with the multiplication on basis elements is defined as follows:

$$
x_{1}^{a_{1}} \cdots x_{n}^{a_{n}} \partial_{u} * x_{1}^{b_{1}} \cdots x_{n}^{b_{n}} \partial_{v}=b_{u} x_{1}^{a_{1}+b_{1}} \cdots x_{n}^{a_{n}+b_{n}} x_{u}^{-1} \partial_{v}
$$

and it can be linearly extended to $N W(n)$ where $\partial_{u}, 1 \leq u \leq n$, is partial derivative of $\mathbb{F}\left[\left[x_{1}, \cdots, x_{n+m}\right]\right]$ with respect to $x_{u}$ (see [6], [7], [8], and [10]). Let us define the $W$-type non-associative algebra $N W(n, m)$ with the standard basis

$$
\left\{e^{a_{1} x_{1}} \cdots e^{a_{n} x_{n}} x_{1}^{u_{1}} \cdots x_{n+m}^{u_{n+m}} \partial_{k} \mid a_{1}, \cdots, a_{n}, u_{1}, \cdots, u_{n+m} \in \mathbb{Z}, 1 \leq k \leq n+m\right\}
$$

and similarly its multiplication $*$ are defined as $(1)$ on its basis elements. We know that the algebra $N W(n)$ is a subalgebra of $N W(n, m)$; please refer to the papers (see [1] and [10]) for more details on a $W$-type algebra. Let $g_{r}$ and $h_{r}, 1 \leq r \leq n$, be additive maps from $\mathbb{Z}$ into $\mathbb{F}$. We define the non-associative algebra $N W\left(g_{n}, h_{n}, n\right)$ with the standard basis

$$
B_{W}=\left\{\left(\begin{array}{c}
a_{1} \\
i_{1}
\end{array}\right) \cdots\left(\begin{array}{c}
a_{n} \\
i_{n}
\end{array}\right) \partial_{k} \mid a_{1}, \cdots, a_{n}, i_{1}, \cdots, i_{n} \in \mathbb{Z}, 1 \leq k \leq n\right\}
$$

and the multiplication $*$ on basis elements is defined as follows:

$$
\begin{aligned}
& \left(\begin{array}{l}
a_{1} \\
i_{1}
\end{array}\right) \cdots\left(\begin{array}{l}
a_{n} \\
i_{n}
\end{array}\right) \partial_{k} *\left(\left(\begin{array}{c}
b_{1} \\
j_{1}
\end{array}\right) \cdots\left(\begin{array}{l}
b_{n} \\
j_{n}
\end{array}\right) \partial_{r}=g_{k}\left(b_{k}\right)\left(\begin{array}{c}
a_{1}+b_{1} \\
i_{1}+j_{1}
\end{array}\right) \cdots\right. \\
& \left(\begin{array}{c}
a_{n}+b_{n} \\
i_{n}+j_{n}
\end{array}\right) \partial_{r}+h_{k}\left(j_{k}\right)\left(\begin{array}{c}
a_{1}+b_{1} \\
i_{1}+j_{1}
\end{array}\right) \cdots\left(\begin{array}{c}
a_{k}+b_{k} \\
i_{k}+j_{k}-1
\end{array}\right)\left(\begin{array}{c}
a_{k+1}+b_{k+1} \\
i_{k+1}+j_{k+1}
\end{array}\right) \cdots \\
& \left(\begin{array}{c}
a_{n}+b_{n} \\
i_{n}+j_{n}
\end{array}\right) \partial_{r}
\end{aligned}
$$

and it can be linearly extended to the algebra $N W\left(g_{n}, h_{n}, n\right)$ (see [2], [3], [7], [8], and [10]). Also, it is not hard to show that the above bracket satisfies the Jacobi identity with respect to the commutator of the algebra (see [4] and [5]). Throughout the paper, $A_{r}, 1 \leq r \leq n$, denotes an additive subgroup of $\mathbb{F}$ or $\mathbb{F}_{p}$. If $h_{r}, 1 \leq r \leq n$, are inclusions, then the algebra $N W\left(g_{n}, h_{n}, n\right)$ is the algebra $N W\left(h_{n}, n\right)$ in the paper (see [10]). Similarly to $N W\left(g_{n}, h_{n}, n\right)$ (resp. 
$\left.N W\left(g_{n}, h_{n}, n\right)^{-}\right)$, we can define the algebra $N W\left(g_{n}, h_{n}, n\right)_{p}\left(\operatorname{resp} . N W\left(g_{n}, h_{n}, n\right)^{-}{ }_{p}\right)$ over $\mathbb{F}_{p}$ by taking the standard basis

$$
B_{W p}=\left\{\left(\begin{array}{c}
a_{1} \\
i_{1}
\end{array}\right) \cdots\left(\begin{array}{c}
a_{n} \\
i_{n}
\end{array}\right) \partial_{k} \mid a_{1}, \cdots, a_{n}, i_{1}, \cdots, i_{n} \in \mathbb{Z}_{p}, 1 \leq k \leq n\right\}
$$

and appropriate mappings $g_{r}$ and $h_{r}$ from $A_{r}$ to $\mathbb{F}_{p}, 1 \leq r \leq n$, where $A_{r}$, $1 \leq r \leq n$, are additive subgroups of $\mathbb{F}_{p}$. Similarly, for additive maps $g_{r}$ and $h_{r}, 1 \leq r \leq n$, from $A_{r}$ to $\mathbb{F}$, then we are able to the $H$-type non-associative algebra $N H\left(g_{2 n}, h_{2 n}, 2 n\right)$ with the standard basis

$$
B_{H}=\left\{\left(\left(\begin{array}{c}
a_{11} \\
i_{11}
\end{array}\right) \cdots\left(\begin{array}{c}
a_{1 n} \\
i_{1 n}
\end{array}\right)\right)\left(\left(\begin{array}{c}
b_{11} \\
j_{11}
\end{array}\right) \cdots\left(\begin{array}{c}
b_{1 n} \\
j_{1 n}
\end{array}\right)\right) \mid a_{11}, \cdots, b_{1 n}, i_{11}, \cdots, j_{1 n} \in \mathbb{Z}\right\}
$$

and the multiplication $*$ is defined as follows:

$$
\begin{aligned}
& \left(\left(\begin{array}{c}
a_{11} \\
i_{11}
\end{array}\right) \cdots\left(\begin{array}{c}
a_{1 n} \\
i_{1 n}
\end{array}\right)\right)\left(\left(\begin{array}{c}
b_{11} \\
j_{11}
\end{array}\right) \cdots\left(\begin{array}{c}
b_{1 n} \\
j_{1 n}
\end{array}\right)\right) *\left(\left(\begin{array}{c}
a_{21} \\
i_{21}
\end{array}\right) \cdots\left(\begin{array}{c}
a_{2 n} \\
i_{2 n}
\end{array}\right)\right) \\
& \left(\left(\begin{array}{c}
b_{21} \\
j_{21}
\end{array}\right) \cdots\left(\begin{array}{c}
b_{2 n} \\
j_{2 n}
\end{array}\right)\right)=\sum_{k=1}^{n} g_{k}\left(a_{1 k}\right) g_{k}\left(b_{2 k}\right)\left(\left(\begin{array}{c}
a_{11}+a_{21} \\
i_{11}+i_{21}
\end{array}\right) \cdots\left(\begin{array}{c}
a_{1 n}+a_{2 n} \\
i_{1 n}+i_{2 n}
\end{array}\right)\right) \\
& \left(\left(\begin{array}{c}
b_{11}+b_{21} \\
j_{11}+j_{21}
\end{array}\right) \cdots\left(\begin{array}{c}
b_{1 n}+b_{2 n} \\
j_{1 n}+j_{2 n}
\end{array}\right)\right)+\sum_{k=1}^{n} g_{k}\left(a_{1 k}\right) h_{k}\left(j_{2 k}\right)\left(\left(\begin{array}{c}
a_{11}+a_{21} \\
i_{11}+i_{21}
\end{array}\right) \cdots\right. \\
& \left.\left(\begin{array}{c}
a_{1 n}+a_{2 n} \\
i_{1 n}+i_{2 n}
\end{array}\right)\right)\left(\left(\begin{array}{c}
b_{11}+b_{21} \\
j_{11}+j_{21}
\end{array}\right) \cdots\left(\begin{array}{c}
b_{1 k}+b_{2 k} \\
j_{1 k}+j_{2 k}-1
\end{array}\right)\left(\begin{array}{c}
b_{1, k+1}+b_{2, k+1} \\
j_{1, k+1}+j_{2, k+1}
\end{array}\right) \cdots\right. \\
& \left.\left(\begin{array}{c}
b_{1 n}+b_{2 n} \\
j_{1 n}+j_{2 n}
\end{array}\right)\left(\begin{array}{l}
b_{1 n}+b_{2 n} \\
j_{1 n}+j_{2 n}
\end{array}\right)\right) \\
& +\sum_{k=1}^{n} h_{k}\left(i_{1 k}\right) g_{k}\left(b_{2 k}\right)\left(\left(\begin{array}{c}
a_{11}+a_{21} \\
i_{11}+i_{21}
\end{array}\right) \cdots\left(\begin{array}{c}
a_{1 k}+a_{2 k} \\
i_{1 k}+i_{2 k}-1
\end{array}\right)\right. \\
& \left.\left(\begin{array}{c}
a_{1, k+1}+a_{2, k+1} \\
i_{1, k+1}+i_{2, k+1}
\end{array}\right) \cdots\left(\begin{array}{c}
a_{1 n}+a_{2 n} \\
i_{1 n}+i_{2 n}
\end{array}\right)\right)\left(\left(\begin{array}{c}
b_{11}+b_{21} \\
j_{11}+j_{21}
\end{array}\right) \cdots\left(\begin{array}{c}
b_{1 n}+b_{2 n} \\
j_{1 n}+j_{2 n}
\end{array}\right)\right) \\
& +\sum_{k=1}^{n} h_{k}\left(a_{1 k}\right) h_{k}\left(j_{2 k}\right)\left(\left(\begin{array}{c}
a_{11}+a_{21} \\
i_{11}+i_{21}
\end{array}\right) \cdots\left(\begin{array}{c}
a_{1 k}+a_{2 k} \\
i_{1 k}+i_{2 k}-1
\end{array}\right)\right. \\
& \left.\left(\begin{array}{c}
a_{1, k+1}+a_{2, k+1} \\
i_{1, k+1}+i_{2, k+1}
\end{array}\right) \cdots\left(\begin{array}{c}
a_{1 n}+a_{2 n} \\
i_{1 n}+i_{2 n}
\end{array}\right)\right)\left(\left(\begin{array}{c}
b_{11}+b_{21} \\
j_{11}+j_{21}
\end{array}\right) \cdots\left(\begin{array}{c}
b_{1 k}+b_{2 k} \\
j_{1 k}+j_{2 k}-1
\end{array}\right)\right. \\
& \left.\left(\begin{array}{c}
b_{1, k+1}+b_{2, k+1} \\
j_{1, k+1}+j_{2, k+1}
\end{array}\right) \cdots\left(\begin{array}{c}
b_{1 n}+b_{2 n} \\
j_{1 n}+j_{2 n}
\end{array}\right)\left(\begin{array}{l}
b_{1 n}+b_{2 n} \\
j_{1 n}+j_{2 n}
\end{array}\right)\right)
\end{aligned}
$$

It is not hard to check that the Jacobi identity holds with respect to the commutator of the algebra $H\left(g_{2 n}, h_{2 n}, 2 n\right)$ (see [10]). Thus the algebra is 
Lie admissible. Since $N H\left(g_{2 n}, h_{2 n}, 2 n\right)$ has the 1-dimensional maximal ideal $<1>$ generated by 1 , we define the quotient algebra $\overline{N H\left(g_{2 n}, h_{2 n}, 2 n\right)}=$ $\frac{N H\left(g_{2 n}, h_{2 n}, 2 n\right)}{<1>}$. From now on, all the maps $g_{k}$ and $h_{l}, 1 \leq k, l \leq n$, will be injective for the simplicity of the algebra $\overline{H\left(g_{2 n}, h_{2 n}, 2 n\right)}$. Similarly, we can define the anti-symmetric algebra $\overline{H\left(g_{2 n}, h_{2 n}, 2 n\right)}$. Similarly to $N H\left(g_{2 n}, h_{2 n}, 2 n\right)$ (resp. $\left.N H\left(g_{2 n}, h_{2 n}, 2 n\right)^{-}\right)$, we can define the algebra $N H\left(g_{2 n}, h_{2 n}, 2 n\right)_{p}$ (resp. $\left.N H\left(g_{2 n}, h_{2 n}, 2 n\right)_{p}{ }^{-}\right)$over $\mathbb{F}_{p}$ with the standard basis

$$
B_{H p}=\left\{\left(\left(\begin{array}{c}
a_{11} \\
i_{11}
\end{array}\right) \cdots\left(\begin{array}{c}
a_{1 n} \\
i_{1 n}
\end{array}\right)\right)\left(\left(\begin{array}{c}
b_{11} \\
j_{11}
\end{array}\right) \cdots\left(\begin{array}{c}
b_{1 n} \\
j_{1 n}
\end{array}\right)\right) \quad \begin{array}{c}
a_{11}, \cdots, b_{1 n}, i_{11}, \cdots, j_{1 n} \\
\left.\in \mathbb{Z}_{p}\right\}
\end{array}\right.
$$

and appropriate mappings $g_{r}$ and $h_{r}$ from $A_{r}$ to $\mathbb{F}_{p}, 1 \leq r \leq n$. Throughout the paper, given maps $g_{r}$ and $h_{r}$ will be additive and injective maps.

\section{$2 \quad$ Simplicity of $N W\left(g_{n}, h_{n}, n\right)$}

The algebra $N W\left(g_{n}, h_{n}, n\right)$ is $\mathbb{Z}^{n}$-graded as follows:

$$
N W\left(g_{r}, h_{r}, n\right)=\bigoplus_{\left(a_{1}, \cdots, a_{n}\right) \in \mathbb{Z}^{n}} N W_{\left(a_{1}, \cdots, a_{n}\right)}
$$

where $N W_{\left(a_{1}, \cdots, a_{n}\right)}$ is the vector subspace of $N W\left(g_{n}, h_{n}, n\right)$ with the standard basis

$$
\bar{B}=\left\{\left(\begin{array}{c}
a_{1} \\
i_{1}
\end{array}\right) \cdots\left(\begin{array}{c}
a_{n} \\
i_{n}
\end{array}\right) \partial_{u} \mid a_{1}, \cdots, a_{n}, i_{1}, \cdots, i_{n} \in \mathbb{Z}, 1 \leq u \leq n\right\}
$$

Let $N W_{\left(a_{1}, \cdots, a_{n}\right)}$ denote the $\left(a_{1}, \cdots, a_{n}\right)$-homogeneous component of $N W\left(g_{n}, h_{n}, n\right)$ and refer to the elements in $N W_{\left(a_{1}, \cdots, a_{n}\right)}$ as $\left(a_{1}, \cdots, a_{n}\right)$-homogeneous elements. Note that the $(0, \cdots, 0)$-homogeneous component is isomorphic to the algebra $N W(n)$. From now on the $(0, \cdots, 0)$-homogeneous component will be called the 0-homogeneous component. Using the lexicographic order on the set $\mathbb{Z}^{2 n} \times\{1, \cdots, n\}$, we introduce the order $>_{o}$ of basis elements of $N W\left(g_{n}, h_{n}, n\right)$ as follows : for any two basis elements $\left(\begin{array}{c}a_{1} \\ i_{1}\end{array}\right) \cdots\left(\begin{array}{c}a_{n} \\ i_{n}\end{array}\right) \partial_{u}$ and $\left(\begin{array}{c}b_{1} \\ j_{1}\end{array}\right) \cdots\left(\begin{array}{c}b_{n} \\ j_{n}\end{array}\right) \partial_{k}$ of $N W\left(g_{n}, h_{n}, n\right)$, we define the order $>_{o}$ as follows: if $\left(a_{1}, \cdots, a_{n}, i_{1}, \cdots, i_{n}, \partial_{u}\right)>\left(b_{1}, \cdots, b_{n}, j_{1}, \cdots, j_{n}, \partial_{k}\right)$, then

$$
\left(\begin{array}{c}
a_{1} \\
i_{1}
\end{array}\right) \cdots\left(\begin{array}{l}
a_{n} \\
i_{n}
\end{array}\right) \partial_{u}>_{o}\left(\begin{array}{l}
b_{1} \\
j_{1}
\end{array}\right) \cdots\left(\begin{array}{c}
b_{n} \\
j_{n}
\end{array}\right) \partial_{k}
$$


where $\partial_{u}>_{o} \partial_{k}$ if $u>k$. Thus we can naturally define the order $>_{o}$ on $N W\left(g_{n}, h_{n}, n\right)$. For any element $l \in N W\left(g_{n}, h_{n}, n\right), l$ can be written as follows using the order and the gradation:

$$
\begin{aligned}
l= & \sum_{i_{1}, \cdots, i_{n}, p} C\left(i_{1}, \cdots, i_{n}, p\right)\left(\begin{array}{c}
a_{11} \\
i_{1}
\end{array}\right) \cdots\left(\begin{array}{c}
a_{1 n} \\
i_{n}
\end{array}\right)_{p}+\cdots \\
& +\sum_{j_{1}, \cdots, j_{n}, q} C\left(j_{1}, \cdots, j_{n}, q\right)\left(\begin{array}{c}
a_{t 1} \\
j_{1}
\end{array}\right) \cdots\left(\begin{array}{c}
a_{t n} \\
j_{n}
\end{array}\right)_{q}
\end{aligned}
$$

where $C\left(i_{1}, \cdots, i_{n}, p\right), \cdots, C\left(j_{1}, \cdots, j_{n}, q\right) \in \mathbb{F}, 1 \leq p, \cdots, q \leq n$. Next, we define the string number $s t(l)$ of $l$ as the number of distinct homogeneous components of $l$ (see $[5,6])$, and $l_{p}(l)$ as $\max \left\{i_{1}, \cdots, i_{n}, \cdots, j_{1}, \cdots, j_{n}\right\}$. For any basis element $\left(\begin{array}{c}a_{1} \\ i_{1}\end{array}\right) \cdots\left(\begin{array}{c}a_{n} \\ i_{n}\end{array}\right)_{r}$ in $\bar{B}$, let us refer to $a_{1}, \cdots, a_{n}$ as the upper indices and $i_{1}, \cdots, i_{n}$ as the lower indices of $\left(\begin{array}{l}a_{1} \\ i_{1}\end{array}\right) \cdots\left(\begin{array}{l}a_{n} \\ i_{n}\end{array}\right)$.

Remark 2.1 If $g_{r}$ and $h_{r}, 1 \leq r \leq n$, are inclusions, then $N W\left(g_{n}, h_{n}, n\right)^{-}$is

the anti-symmetric (i.e., Lie) algebra which is studied in the paper (see [8]).

Lemma 1 For a non-zero element $l$ of $N W\left(g_{n}, h_{n}, n\right)$, the ideal $<l>$ generated by $l$ contains an element such that its all the lower indices are positive.

Proof. Let $l$ be a nonzero element of $N W\left(g_{n}, h_{n}, n\right)$ such that $\left(\begin{array}{l}a_{1} \\ i_{1}\end{array}\right) \cdots\left(\begin{array}{l}a_{n} \\ i_{n}\end{array}\right)_{u}$ is the maximal term of $l$ with respect to the order $>_{o}$. We can assume that either $a_{1}$ or $i_{1}$ is non-zero scalar. If we take an element $l_{1}=\left(\begin{array}{c}0 \\ j_{1}\end{array}\right) \cdots\left(\begin{array}{c}0 \\ j_{n}\end{array}\right)_{t}$ such that $j_{1}>>\cdots>>j_{n}>>0$, then $l_{1} * l \neq 0$ is the required element of the lemma where $a>>b$ means $a$ is sufficiently larger than $b$.

Lemma 2 Let $I$ be an ideal of the algebra $N W\left(g_{n}, h_{n}, n\right)$. If I contains an element $\left(\begin{array}{l}0 \\ 0\end{array}\right) \cdots\left(\begin{array}{l}0 \\ 0\end{array}\right)_{u}, 1 \leq u \leq n$, then $I=N W\left(g_{n}, h_{n}, n\right)$.

Proof. Since $N W(n)$ is a simple subalgebra $N W\left(g_{n}, h_{n}, n\right)$ of the algebra $N W\left(g_{n}, h_{n}, n\right)$, for any element $l \in N W(n)$, the ideal $<l>$ contains $N W(n)$ where $\langle l\rangle$ is an ideal of $N W\left(g_{n}, h_{n}, n\right)$ and it is generated by the element $l$. Let $I$ be a non-zero ideal of $N W\left(g_{n}, h_{n}, n\right)$ in the lemma. Let $\left(\begin{array}{c}0 \\ j_{1}\end{array}\right) \cdots\left(\begin{array}{c}0 \\ j_{n}\end{array}\right)_{t}$ be the element in the lemma. Thus the ideal $<\left(\begin{array}{c}0 \\ j_{1}\end{array}\right) \cdots\left(\begin{array}{c}0 \\ j_{n}\end{array}\right)_{t}>$ of $N W\left(g_{n}, h_{n}, n\right)$ contains the algebra $N W(n)$ where $<\left(\begin{array}{c}0 \\ j_{1}\end{array}\right) \cdots\left(\begin{array}{c}0 \\ j_{n}\end{array}\right)_{t}>$ is the ideal generated by the element $\left(\begin{array}{c}0 \\ j_{1}\end{array}\right) \cdots\left(\begin{array}{c}0 \\ j_{n}\end{array}\right)$. Let $\left(\begin{array}{c}a_{1} \\ i_{1}\end{array}\right)\left(\begin{array}{c}a_{2} \\ i_{2}\end{array}\right) \cdots\left(\begin{array}{c}a_{n} \\ i_{n}\end{array}\right) \partial_{u}$ be any basis element of in the 
standard basis of $N W\left(g_{n}, h_{n}, n\right)$. Without loss of generality, we can assume that $a_{1} \neq 0$. If $i_{1}=0$, the we have that

$$
\begin{gathered}
\left(\begin{array}{c}
0 \\
m
\end{array}\right)\left(\begin{array}{l}
0 \\
0
\end{array}\right) \cdots\left(\begin{array}{l}
0 \\
0
\end{array}\right) \partial_{1} *\left(\begin{array}{c}
a_{1} \\
0
\end{array}\right)\left(\begin{array}{c}
a_{2} \\
i_{2}
\end{array}\right) \cdots\left(\begin{array}{c}
a_{n} \\
i_{n}
\end{array}\right) \partial_{t} \\
=g_{1}\left(a_{1}\right)\left(\begin{array}{l}
a_{1} \\
m
\end{array}\right)\left(\begin{array}{c}
a_{2} \\
i_{2}
\end{array}\right) \cdots\left(\begin{array}{c}
a_{n} \\
i_{n}
\end{array}\right) \partial_{t}
\end{gathered}
$$

Since $g_{1}\left(a_{1}\right) \neq 0$, we have that $\left(\begin{array}{c}a_{1} \\ 0\end{array}\right)\left(\begin{array}{c}a_{2} \\ i_{2}\end{array}\right) \cdots\left(\begin{array}{c}a_{n} \\ i_{n}\end{array}\right) \partial_{t} \in I$. This implies that by induction on $m \in \mathbb{N}$ of $\left(\begin{array}{c}a_{1} \\ m\end{array}\right)\left(\begin{array}{c}a_{2} \\ i_{2}\end{array}\right) \cdots\left(\begin{array}{c}a_{n} \\ i_{n}\end{array}\right) \partial_{t}$, we can prove that $\left(\begin{array}{c}a_{1} \\ i_{1}\end{array}\right)\left(\begin{array}{c}a_{2} \\ i_{2}\end{array}\right) \cdots\left(\begin{array}{c}a_{n} \\ i_{n}\end{array}\right) \partial_{t} \in$ $I$ where $i_{1} \in \mathbb{N}$. Similarly, by induction on $m \in \mathbb{Z}$ of $\left(\begin{array}{c}a_{1} \\ m\end{array}\right)\left(\begin{array}{c}a_{2} \\ i_{2}\end{array}\right) \cdots\left(\begin{array}{c}a_{n} \\ i_{n}\end{array}\right) \partial_{t}$, we can also prove that $\left(\begin{array}{c}a_{1} \\ i_{1}\end{array}\right)\left(\begin{array}{c}a_{2} \\ i_{2}\end{array}\right) \cdots\left(\begin{array}{c}a_{n} \\ i_{n}\end{array}\right) \partial_{t} \in I$ where $i_{1} \in \mathbb{Z}$. Therefore we have proven the lemma.

Theorem 1 The algebra $N W\left(g_{n}, h_{n}, n\right)$ is simple.

Proof. Let $I$ be any non-zero ideal of $N W\left(g_{n}, h_{n}, n\right)$. Note that $N W\left(g_{n}, h_{n}, n\right)$ is $\mathbb{Z}^{n}$-graded and we have the following non-zero element in $N W_{0, \cdots, 0}$

$$
\left(\begin{array}{l}
a_{1} \\
i_{1}
\end{array}\right) \cdots\left(\begin{array}{c}
a_{n} \\
i_{n}
\end{array}\right) \partial_{r} *\left(\begin{array}{c}
-a_{1} \\
j_{1}
\end{array}\right) \cdots\left(\begin{array}{c}
-a_{n} \\
j_{n}
\end{array}\right) \partial_{s} \in N W_{0, \cdots, 0}
$$

where $i_{1}, \cdots, i_{n}, j_{1}, \cdots, j_{n} \in \mathbb{Z}$ and $N W_{0, \cdots, 0}$ is the $(0, \cdots, 0)$-homogeneous component of $N W\left(g_{n}, h_{n}, n\right)$. Let $l$ be a non-zero element of $I$. Let us prove the theorem by induction on $s t(l)$ of the element $l$. If $s t(l)=1$, then there is nothing to prove by (8) and Lemma 2. If $s t(l)=k \geq 1$, then by induction we can assume that $N W\left(g_{n}, h_{n}, n\right)=<l>=I$. Let us assume that $s t(l)=$ $k+1$. Either $l$ has a term in the $N W_{(0, \cdots, 0)}$ homogeneous component or not, by Lemma 2 and (8), we have an element $l_{1}$ of $<l>$ such that $\operatorname{st}\left(l_{1}\right)<k+1$. This implies that by induction, $N W\left(g_{n}, h_{n}, n\right)=<l_{1}>\subset I$, i.e., $N W\left(g_{n}, h_{n}, n\right)=I$. This implies that $N W\left(g_{n}, h_{n}, n\right)$ is simple. Therefore we have proven the theorem.

Corollary 1 The algebra $N W(n, 0)$ is simple.

Proof. If we take additive embedding $g_{r}, \quad h_{r}: \mathbb{Z}$ to $\mathbb{F}, 1 \leq r \leq n$, then we get the required results of the corollary (see [1] and [10]).

Corollary 2 The anti-symmetric algebra $N W\left(g_{n}, h_{n}, n\right)^{-}$is simple.

Proof. The proof of the well known (see [10]), but it is also straightforward by the similar proof of Theorem 1 . So it is omitted. 
Theorem 2 The algebra $N W\left(g_{n}, h_{n}, n\right)_{p}$ (resp. $\left.N W\left(g_{n}, h_{n}, n\right)_{p}{ }^{-}\right)$is simple.

Proof. The proof of the theorem is similar to the proof of Theorem 1 and Corollary 1, so omitted.

It is an interesting problem to find all the automorphisms of the subalgebra $N W(h, 1)$ of the algebra $N W\left(h_{n}, n\right)$.

Theorem 3 For any algebra automorphism $\theta \in \operatorname{Aut}(N W(h, 1))$, we have that

$$
\theta\left(\left(\begin{array}{l}
0 \\
1
\end{array}\right)_{1}\right)=\sum_{j} C_{j}\left(\begin{array}{l}
0 \\
j
\end{array}\right)_{1}
$$

where $C_{j} \in F$.

Proof. Let $\theta$ be the automorphism of $N W(h, 1)$ in the lemma. Note that for any elements $l_{1} \in N W_{a}$ and $l_{2} \in N W_{b}$ such that $a \neq b$, we have that $l_{1} * l_{2} \neq 0$. Since $\left(\begin{array}{l}0 \\ 1\end{array}\right)$, is a right identity element of $N W_{0}$ with respect to the standard basis, the theorem follows from the above comment and the fact that $\theta$ is a homomorphism.

It is easy to prove that there is an injective algebra homomorphism $\theta$ of $N W(g, h, 1)$ such that $\theta$ is not surjective. Thus every non-zero endomorphism of $N W(g, h, 1)$ is not an automorphism of $N W(g, h, 1)$. An element $l \in N W\left(g_{n}, h_{n}, n\right)$ is left stable (resp. right stable) with respect to a basis $B$ of $N W\left(g_{n}, h_{n}, n\right)$, if $l * m=\alpha(m) m$ (resp. $m * l=\alpha(m) m$ ) for all $m \in B$ where $\alpha(m)$ is a scalar depending on $l$ and $m$.

\section{$3 \quad H$-type Algebras}

In this section, we prove that the algebra $\overline{N H\left(g_{2 n}, h_{2 n}, 2 n\right)}$ is simple. The Lie algebra $\overline{N H\left(g_{2 n}, h_{2 n}, 2 n\right)}$ is $\mathbb{Z}^{2 n}$-graded as follows:

$$
\overline{N H\left(g_{2 n}, h_{2 n}, 2 n\right)}=\bigoplus_{\left(a_{1}, \cdots, a_{n}, b_{1}, \cdots, b_{n}\right) \in \mathbb{Z}^{2 n}} N H_{\left(a_{1}, \cdots, a_{n}, b_{1}, \cdots, b_{n}\right)}
$$

where $N H_{\left(a_{1}, \cdots, a_{n}, b_{1}, \cdots, b_{n}\right)}$ is the $\left(a_{1}, \cdots, a_{n}, b_{1}, \cdots, b_{n}\right)$-homogeneous component of $\overline{N H\left(g_{2 n}, h_{2 n}, 2 n\right)}$ as $N W\left(h_{p}, g_{p}, n\right)$ (see Section 2). Using the commutator of $N H\left(g_{2 n}, h_{2 n}, 2 n\right)$, we are able to define the anti-symmetric algebra $N H\left(g_{2 n}, h_{2 n}, 2 n\right)^{-}$and its subalgebra $\overline{N H\left(g_{2 n}, h_{2 n}, 2 n\right)}-$. Note that these antisymmetric algebras are Lie algebras. 
Lemma 3 If $l \in \overline{N H\left(g_{2 n}, h_{2 n}, 2 n\right)}$ is a non-zero element, then the ideal $<l>$ generated by the element $l$ contains an element $l_{1}$ whose lower indices are positive integers.

Proof. Let $l$ be the element in the lemma. The element

$$
l *\left(\left(\begin{array}{c}
0 \\
s_{1}
\end{array}\right)\left(\begin{array}{c}
0 \\
s_{2}
\end{array}\right) \cdots\left(\begin{array}{c}
0 \\
s_{n}
\end{array}\right)\right)\left(\left(\begin{array}{c}
0 \\
t_{1}
\end{array}\right) \cdots\left(\begin{array}{c}
0 \\
t_{n}
\end{array}\right)\right)
$$

is the required non-zero element in the lemma, where we took $s_{1}>>\cdots>>$ $s_{n}>>t_{1}>>\cdots>>t_{n}>>0$ and $a>>b$ means that $a$ is a sufficiently large number than $b$. Therefore, we have proven the lemma.

Lemma 4 If an ideal I of $\overline{N H\left(g_{2 n}, h_{2 n}, 2 n\right)}$ contains $\delta_{1}:=\left(\left(\begin{array}{l}0 \\ 1\end{array}\right)\left(\begin{array}{l}0 \\ 0\end{array}\right) \cdots\left(\begin{array}{l}0 \\ 0\end{array}\right)\right)$ $\left(\left(\begin{array}{l}0 \\ 0\end{array}\right) \cdots\left(\begin{array}{l}0 \\ 0\end{array}\right)\right)$ or, $\cdots$, or, $\delta_{2 n}:=\left(\left(\begin{array}{l}0 \\ 0\end{array}\right) \cdots\left(\begin{array}{l}0 \\ 0\end{array}\right)\right)\left(\left(\begin{array}{l}0 \\ 0\end{array}\right) \cdots\left(\begin{array}{l}0 \\ 0\end{array}\right)\left(\begin{array}{l}0 \\ 1\end{array}\right)\right)$, then $I=\overline{N H\left(g_{2 n}, h_{2 n}, 2 n\right)}$.

Proof. Let $I$ be the ideal of $\overline{N H\left(g_{2 n}, h_{2 n}, 2 n\right)}$ in the lemma. Without loss of generality, we can assume that $I$ contains $\delta_{1}=\left(\left(\begin{array}{l}0 \\ 1\end{array}\right)\left(\begin{array}{l}0 \\ 0\end{array}\right) \cdots\left(\begin{array}{l}0 \\ 0\end{array}\right)\right)\left(\left(\begin{array}{l}0 \\ 0\end{array}\right) \cdots\left(\begin{array}{l}0 \\ 0\end{array}\right)\right)$. Since $g_{i}$, and $h_{i}$ are one to one mappings, $1 \leq i \leq n$, we have that

$$
\left\{\left(\left(\begin{array}{c}
0 \\
i_{1}
\end{array}\right)\left(\begin{array}{c}
0 \\
i_{2}
\end{array}\right) \cdots\left(\begin{array}{c}
0 \\
i_{n}
\end{array}\right)\right)\left(\left(\begin{array}{c}
0 \\
j_{1}
\end{array}\right) \cdots\left(\begin{array}{c}
0 \\
j_{n}
\end{array}\right)\right) \mid i_{1}, \cdots, i_{n}, j_{1}, \cdots, j_{n} \in \mathbb{Z}\right\} \subset I .
$$

Let us take an element $\left(\begin{array}{c}a_{1} \\ i_{1}\end{array}\right)\left(\begin{array}{c}a_{2} \\ i_{2}\end{array}\right) \cdots\left(\begin{array}{c}a_{n} \\ i_{n}\end{array}\right)\left(\left(\begin{array}{l}b_{1} \\ j_{1}\end{array}\right) \cdots\left(\begin{array}{c}b_{1} \\ j_{n}\end{array}\right)\right)$ of $\overline{N H\left(g_{2 n}, h_{2 n}, 2 n\right)}$ such that $b_{1} \neq 0$. Then we have that

$$
\begin{aligned}
& \delta_{1} *\left(\left(\begin{array}{c}
a_{1} \\
i_{1}
\end{array}\right)\left(\begin{array}{c}
a_{2} \\
i_{2}
\end{array}\right) \cdots\left(\begin{array}{l}
a_{n} \\
i_{n}
\end{array}\right)\right)\left(\left(\begin{array}{l}
b_{1} \\
j_{1}
\end{array}\right) \cdots\left(\begin{array}{l}
b_{1} \\
j_{n}
\end{array}\right)\right) \\
= & g_{n+1}\left(b_{1}\right)\left(\left(\begin{array}{c}
a_{1} \\
i_{1}
\end{array}\right)\left(\begin{array}{c}
a_{2} \\
i_{2}
\end{array}\right) \cdots\left(\begin{array}{c}
a_{n} \\
i_{n}
\end{array}\right)\right)\left(\left(\begin{array}{c}
b_{1} \\
j_{1}
\end{array}\right) \cdots\left(\begin{array}{l}
b_{1} \\
j_{n}
\end{array}\right)\right) \\
+ & h_{n+1}\left(j_{1}\right)\left(\left(\begin{array}{c}
a_{1} \\
i_{1}
\end{array}\right)\left(\begin{array}{c}
a_{2} \\
i_{2}
\end{array}\right) \cdots\left(\begin{array}{c}
a_{n} \\
i_{n}
\end{array}\right)\right)\left(\left(\begin{array}{c}
b_{1} \\
j_{1}-1
\end{array}\right) \cdots\left(\begin{array}{l}
b_{1} \\
j_{n}
\end{array}\right)\right) \in I
\end{aligned}
$$

By taking $j_{1}=0$ in (9), we also have that

$$
\left(\left(\begin{array}{c}
a_{1} \\
i_{1}
\end{array}\right)\left(\begin{array}{c}
a_{2} \\
i_{2}
\end{array}\right) \cdots\left(\begin{array}{c}
a_{n} \\
i_{n}
\end{array}\right)\right)\left(\left(\begin{array}{c}
b_{1} \\
0
\end{array}\right) \cdots\left(\begin{array}{l}
b_{1} \\
j_{n}
\end{array}\right)\right) \in I
$$

for all $a_{1}, \cdots, a_{n}, b_{1}, \cdots, b_{n}, i_{1}, \cdots, i_{n}, j_{2}, \cdots, j_{n} \in \mathbb{Z}$. Let us prove this lemma by induction on $j_{1}$ of the element $\left(\left(\begin{array}{c}a_{1} \\ i_{1}\end{array}\right)\left(\begin{array}{c}a_{2} \\ i_{2}\end{array}\right) \cdots\left(\begin{array}{c}a_{n} \\ i_{n}\end{array}\right)\right)\left(\left(\begin{array}{c}b_{1} \\ j_{1}\end{array}\right) \cdots\left(\begin{array}{c}b_{1} \\ j_{n}\end{array}\right)\right)$. For $j_{1}$, we can assume that $\left(\left(\begin{array}{c}a_{1} \\ i_{1}\end{array}\right)\left(\begin{array}{c}a_{2} \\ i_{2}\end{array}\right) \cdots\left(\begin{array}{c}a_{n} \\ i_{n}\end{array}\right)\right)\left(\left(\begin{array}{c}b_{1} \\ j_{1}-1\end{array}\right) \cdots\left(\begin{array}{c}b_{1} \\ j_{n}\end{array}\right)\right) \in I$. This implies that the element $\left(\left(\begin{array}{c}a_{1} \\ i_{1}\end{array}\right)\left(\begin{array}{c}a_{2} \\ i_{2}\end{array}\right) \cdots\left(\begin{array}{c}a_{n} \\ i_{n}\end{array}\right)\right)\left(\left(\begin{array}{c}b_{1} \\ j_{1}\end{array}\right) \cdots\left(\begin{array}{c}b_{1} \\ j_{n}\end{array}\right)\right)$ is in the ideal $I$ from $(9)$. For $j_{1}<0$, if 
we assume that $\left(\left(\begin{array}{c}a_{1} \\ i_{1}\end{array}\right)\left(\begin{array}{c}a_{2} \\ i_{2}\end{array}\right) \cdots\left(\begin{array}{c}a_{n} \\ i_{n}\end{array}\right)\right)\left(\left(\begin{array}{c}b_{1} \\ j_{1}\end{array}\right) \cdots\left(\begin{array}{c}b_{1} \\ j_{n}\end{array}\right)\right)$ is in the ideal $I$, then by (9), we can prove that $\left(\left(\begin{array}{c}a_{1} \\ i_{1}\end{array}\right)\left(\begin{array}{c}a_{2} \\ i_{2}\end{array}\right) \cdots\left(\begin{array}{c}a_{n} \\ i_{n}\end{array}\right)\right)\left(\left(\begin{array}{c}b_{1} \\ j_{1}-1\end{array}\right) \cdots\left(\begin{array}{c}b_{1} \\ j_{n}\end{array}\right)\right) \in I$. Therefore for any $j_{1} \in \mathbb{Z}$, we have that

$\left\{\left(\left(\begin{array}{c}a_{1} \\ i_{1}\end{array}\right)\left(\begin{array}{c}a_{2} \\ i_{2}\end{array}\right) \cdots\left(\begin{array}{c}a_{n} \\ i_{n}\end{array}\right)\right)\left(\left(\begin{array}{c}b_{1} \\ j_{1}\end{array}\right) \cdots\left(\begin{array}{c}b_{1} \\ j_{n}\end{array}\right)\right) \mid a_{1}, \cdots, a_{n}, b_{1}, \cdots, b_{n}, i_{1}, \cdots, i_{n}, j_{2}, \cdots, j_{n} \in \mathbb{Z}\right\}$ is a subset of $I$. This implies that all the basis elements of $\overline{N H\left(g_{2 n}, h_{2 n}, 2 n\right)}$ are in the ideal $I$. This implies $I=\overline{N H\left(g_{2 n}, h_{2 n}, 2 n\right)}$. Therefore we have proven the lemma.

Theorem 4 The algebra $\overline{N H\left(g_{2 n}, h_{2 n}, 2 n\right)}$ is simple.

Proof. Let $I$ be a non-zero ideal of $\overline{N H\left(g_{2 n}, h_{2 n}, 2 n\right)}$. Let $l$ be a non-zero element of $I$. Note that every element $l$ of $\overline{N H\left(g_{2 n}, h_{2 n}, 2 n\right)}$ can be written as the terms in its appropriate components using the order of the algebra. Let us prove this theorem by induction on $s t(l)$ of the element $l$. Let us assume that $l$ is a non-zero element in $I$ such that $s t(l)=1$. If $l$ is the sum of terms in the $(0, \cdots, 0)$-homogeneous component, then we have proven the theorem by Lemma 5 . If $l$ is the sum of terms in the $\left(a_{1}, \cdots, a_{2 n}\right)$-homogeneous component, then

$$
\left(\left(\begin{array}{c}
-a_{1} \\
t_{1}
\end{array}\right)\left(\begin{array}{c}
-a_{2} \\
0
\end{array}\right) \cdots\left(\begin{array}{c}
-a_{n} \\
0
\end{array}\right)\right)\left(\left(\begin{array}{c}
-a_{n+1} \\
0
\end{array}\right) \cdots\left(\begin{array}{c}
-a_{2 n} \\
0
\end{array}\right)\right) * l
$$

is in $I$ such that $l$ is the sum of terms in $(0, \cdots, 0)$-homogeneous component. Since $I$ contains a term of $(0, \cdots, 0)$-homogeneous component, we are able to prove that $I=\overline{N H\left(g_{2 n}, h_{2 n}, 2 n\right)}$. Therefore, we have proven the theorem. Inductively, we can assume that if $l$ is the sum of terms in the $(k)$ homogeneous components, then $I=\overline{N H\left(g_{2 n}, h_{2 n}, 2 n\right)}$. Assume $l$ is the sum of elements in the $(k+1)$ homogeneous components. If $l$ has a term in the $(0, \cdots, 0)$-homogeneous component and $a_{j}, 1 \leq j \leq n$, is the first non-zero upper index and $p$ be the maximal first lower index. We have the following non-zero element

$$
\left.l_{3}=\left(\cdots\left(l * \delta_{j+n}\right) *\right) \delta_{j+n} * \cdots * \delta_{j+n}\right)
$$

and it is in $I$ where we applied $(p+1)$-times of the multiplication in (10). Then the element $l_{3}$ is in $I$ such that it has terms in the $(k)$ homogeneous components. Therefore, we have proven the theorem by induction. If $l$ has no term in the $(0, \cdots, 0)$-homogeneous component and it has a term in the $\left(a_{1}, \cdots, a_{2 n}\right)$-homogeneous component, then the element

$$
l_{4}=\left(\left(\begin{array}{c}
-a_{1} \\
0
\end{array}\right)\left(\begin{array}{c}
-a_{2} \\
0
\end{array}\right) \cdots\left(\begin{array}{c}
-a_{n} \\
0
\end{array}\right)\right)\left(\left(\begin{array}{c}
-a_{n+1} \\
0
\end{array}\right) \cdots\left(\begin{array}{c}
-a_{2 n} \\
0
\end{array}\right)\right) * l
$$

is a non-zero element in $I$ and $l_{4}$ has terms in at most $(k+1)$ homogeneous components. It may have a term in the $(0, \cdots, 0)$-homogeneous component or 
not. But we have already proven theorem on that case. Therefore, we have proven the theorem.

Corollary 3 The anti-symmetric algebra ${\overline{N H\left(g_{2 n}, h_{2 n}, 2 n\right)}}^{-}$is simple.

Proof. The proof of the well known (see [8]), but it is straightforward by Theorem 3.

\left. Theorem 5 The algebra ${\overline{N H\left(g_{2 n}, h_{2 n}, 2 n\right)_{p}}}_{\left(\text {resp. } \overline{N H\left(g_{2 n}, h_{2 n}, 2 n\right)}\right.}{ }_{p}^{-}\right)$is simple.

Proof. The proof of theorem is similar to the proof of Theorem 3 (resp. Corollary 3), so it is omitted.

By reviewing the proofs of Theorem 2 and Theorem 4-5, the algebras $N W\left(g_{p}, h_{p}, n\right)$,

$N W\left(g_{p}, h_{p}, n\right)_{p}, \overline{N H\left(g_{2 n}, h_{2 n}, 2 n\right)}$ and $\overline{N H\left(g_{2 n}, h_{2 n}, 2 n\right)}$ have a class of simple subalgebras by taking appropriate additive monoids instead of integers or $\mathbb{Z}_{p}$ on lower indices in the definitions of the given appropriate algebras.

\section{Open problems on the algebras in the paper}

This is a good place to suggest some open problems on the simple Lie algebras which are defined in this paper to compare with properties of the well-known Lie algebras. The following is related to the Jacobian conjecture on the $W$-type algebra $N W\left(g_{p}, h_{p}, n\right), n>1$.

Question 1. Is every non-zero endomorphism of $N W\left(g_{p}, h_{p}, n\right)$

(resp. $\left.\overline{N H\left(g_{2 n}, h_{2 n}, 2 n\right)}\right)$ is an automorphism of $N W\left(g_{p}, h_{p}, n\right)$ (resp.

$\left.\overline{N H\left(g_{2 n}, h_{2 n}, 2 n\right)}\right)$ ?

The answer is no. Since every non-zero endomorphism of $N W\left(g_{p}, h_{p}, n\right)$ (resp.

$\left.\overline{N H\left(g_{2 n}, h_{2 n}, 2 n\right)}\right)$ is injective, it is enough to show that every non-zero endomorphism is surjective for the Jacobi conjecture on $N W\left(g_{p}, h_{p}, n\right)$ (resp. $\left.\overline{N H\left(g_{2 n}, h_{2 n}, 2 n\right)}\right)$.

Question 2. Find the automorphism group $\operatorname{Aut}\left(N W\left(g_{p}, h_{p}, n\right)\right)$ (resp. Aut $\left.\left(\overline{N H\left(g_{2 n}, h_{2 n}, 2 n\right)}\right)\right)$ of the algebra $N W\left(g_{p}, h_{p}, n\right)$ (resp. $\left.\overline{N H\left(g_{2 n}, h_{2 n}, 2 n\right)}\right)$. 


\section{References}

[1] Mohammad H. Ahmadi, Ki-Bong Nam, and Jonathan Pakinathan, Lie admissible non-associative algebras, Algebra Colloquium, Vol. 12, No. 1, World Scientific, March, (2005), 113-120.

[2] Seul Hee Choi and Ki-Bong Nam, Derivations of a restricted Weyl Type Algebra I, Accepted, Rocky Mountain Journal of Mathematics, (2005).

[3] Seul Hee Choi and Ki-Bong Nam, Weyl type non-associative algebra using additive groups I, Algebra Colloquium, Accepted, (2005).

[4] I. N. Herstein, Topics in Ring Theory, The University of Chicago Press, (1965), 1-10.

[5] J. E. Humphreys, Introduction to Lie Algebras and Representation Theory, Springer-Verlag, New York, 1987.

[6] V. G. Kac, Description of Filtered Lie Algebra with which Graded Lie algebras of Cartan type are Associated, Izv. Akad. Nauk SSSR, Ser. Mat. Tom, 38, (1974), 832-834.

[7] Naoki Kawamoto, Atsushi Mitsukawa, Ki-Bong Nam and Moon-Ok Wang, The automorphisms of generalized Witt type Lie algebras, Journal of Lie Theory, Vol (13) (2003), 571-576.

[8] Ki-Bong Nam, Generalized $W$ and $H$ Type Lie Algebras, Algebra Colloquium 6:3, (1999), 329-340.

[9] Nam, Ki-Bong, Modular $W$ and $H$ type Lie algebras, Southeast Asian Bull. Math. 26 (2002), No. 2, 245-255.

[10] Ki-Bong Nam, On Some Non-Associative Algebras Using Additive Groups, Southeast Asian Bulletin of Mathematics, Vol. 27, Springer Verlag, (2003), 493-500.

[11] Ki-Bong Nam and Seul Hee Choi, On evaluation algebras, Southeast Asian Bull. Math., 29 (2005), No. 2, 381-385. 
[12] R. D. Schafer, Introduction to nonassociative algebras, Dover, 1995, 128138.

Received: November 28, 2006 